Bull. Austral. Math. Soc.

Vol. 45 (1992) [261-266]

\title{
STEINHARDT'S INEQUALITY IN THE MINKOWSKI PLANE
}

\author{
Mostafa GhandehaRI
}

In a Minkowski plane with unit circle $E$, the product of the positive circumference of a plane convex body $K$ and that of its polar dual is greater than or equal to the square of the Euclidean length of the polar dual of $E$. Equality holds if and only if $K$ is a Euclidean unit circle.

\section{INTRODUCTION}

By a plane convex body $K$ we shall mean a compact, convex subset of the Euclidean plane having a non-empty interior. Let $K_{1}$ and $K_{2}$ be plane convex bodies, both having the origin as an interior point. Define $\sigma_{+}\left(K_{1}, K_{2}\right)$ as the length of the positively oriented boundary of $K_{1}$, measured with respect to the (nonsymmetric) metric induced by $K_{2}$. Similarly define $\sigma_{-}\left(K_{1}, K_{2}\right)$ as the length of the negatively oriented boundary of $K_{1}$, measured with respect to $K_{2}$.

The main purpose of this note is to prove that if $E$ is the unit circle of a given Minkowski plane and $K$ is a plane convex body then

$$
\sigma_{+}(K, E) \sigma_{+}\left(K^{*}, E\right) \geqslant\left[L\left(E^{*}\right)\right]^{2},
$$

where $L\left(E^{*}\right)$ denotes the Euclidean length of the polar dual of $E$ and $K^{*}$ denotes the polar dual of $K$. The polar dual is taken with respect to an interior point of $K$. In the case that $E$ is a Euclidean unit circle, Steinhardt's inequality [6] follows:

$$
L(K) L\left(K^{*}\right) \geqslant 4 \pi^{2}
$$

The preliminary definitions and results are given in the next section. The proof of the main result is given in Section 3. Related results are also given in Section 3.

\section{Received 2 April 1991}

The author is thankful to the Naval Postgraduate School for support during this work.

Copyright Clearance Centre, Inc. Serial-fee code: 0004-9729/82 \$A2.00+0.00. 


\section{Preliminary definitions and Results}

Let $K$ be a plane convex body with the origin as an interior point. For each angle $\theta, 0 \leqslant \theta<2 \pi$, we let $r(K, \theta)$ be the radius of $K$ in direction $(\cos \theta, \sin \theta)$, so that the boundary of $K$ has equation $r=r(K, \theta)$ in polar coordinates. The distance from the origin to the supporting line of $K$ with outward normal $(\cos \theta, \sin \theta)$ is noted by $h(K, \theta)$. This is the supporting function of $K$ restricted to the Euclidean unit circle. Since $K$ is convex, it has a well defined unique tangent line at all but at most a countable number of points. We let $d s(K, \theta)$ represent the element of Euclidean arclength of the boundary of $K$ at a point where the unit normal is given by $(\cos \theta, \sin \theta)$. The Euclidean length of $K$ is given by

$$
L(K)=\int_{0}^{2 \pi} h(K, \theta) d \theta
$$

while the Euclidean area of $K$ is given by

$$
A(K)=\frac{1}{2} \int_{0}^{2 \pi} h(K, \theta) d s(K, \theta) .
$$

The polar dual of $K$, denoted by $K^{*}$, is another plane convex body having the origin as an interior point and is defined in such a way that

$$
h\left(K^{*}, \theta\right)=\frac{1}{r(K, \theta)} \text { and } r\left(K^{*}, \theta\right)=\frac{1}{h(K, \theta)} .
$$

The mixed area $A\left(K_{1}, K_{2}\right)$ of two convex sets is defined by

$$
A\left(K_{1}, K_{2}\right)=\frac{1}{2} \int_{0}^{2 \pi} h\left(K_{1}, \theta\right) d s\left(K_{2}, \theta\right) .
$$

It turns out that the mixed area is symmetric in its arguments. Eggleston [2] contains further properties of mixed areas.

If $K$ is a centrally symmetric plane convex body, centred at the origin, then the self-circumference $\sigma(K)$ is given by

$$
\sigma(K)=\int \frac{d s(K, \theta)}{r\left(K, \theta+\frac{\pi}{2}\right)} .
$$

If $K$ is not necessarily symmetric and $z$ is any point interior to $K$, then positive and negative self-circumference of $K$ relative to $z$ are defined by

$$
\sigma_{+}(K, z)=\int \frac{d s(K, \theta)}{r\left(K, \theta+\frac{\pi}{2}\right)}
$$

and

$$
\sigma_{-}(K, z)=\int \frac{d s(K, \theta)}{r\left(K, \theta-\frac{\pi}{2}\right)}
$$


where the origin of the coordinate system is at $z$.

Both $\sigma_{+}(K, z)$ and $\sigma_{-}(K, z)$ reduce to $\sigma(K)$ in case $K$ is centrally symmetric with $z$ as its centre.

If $K_{1}$ and $K_{2}$ are plane convex bodies with the origin as an interior point, then the length of the positively oriented boundary of $K_{1}$ with respect to $K_{2}$ is given by

$$
\sigma_{+}\left(K_{1}, K_{2}\right)=\int \frac{d s\left(K_{1}, \theta\right)}{r\left(K_{2}, \theta+\frac{\pi}{2}\right)}
$$

and the length of the negatively oriented boundary is given by

$$
\sigma_{-}\left(K_{1}, K_{2}\right)=\int \frac{d s\left(K_{1}, \theta\right)}{r\left(K_{2}, \theta-\frac{\pi}{2}\right)}
$$

Schäffer [5] and independently later, Thompson [7], proved that for a centrally symmetric set $\sigma_{+}(K)=\sigma_{-}\left(K^{*}\right)$ and $\sigma_{-}(K)=\sigma_{+}\left(K^{*}\right)$. More generally Chakerian [1] used the concept of mixed areas to prove that

$$
\begin{aligned}
& \sigma_{+}\left(K_{1}, K_{2}\right)=\sigma_{-}\left(K_{2}^{*}, K_{1}^{*}\right) \quad \text { and } \\
& \sigma_{-}\left(K_{1}, K_{2}\right)=\sigma_{+}\left(K_{2}^{*}, K_{1}^{*}\right)
\end{aligned}
$$

The unit circle $E$ of a Minkowski plane is referred to as the indicatrix. Define the isoperimetrix to be that convex body $T$ such that

$$
h(T, \theta)=\frac{1}{r\left(E, \theta+\frac{\pi}{2}\right)}=h\left(E^{*}, \theta+\frac{\pi}{2}\right) .
$$

A centrally symmetric set is called a Radon curve if it coincides with the corresponding isoperimetrix. In the next section we use properties of mixed areas to discuss self-circumference of Radon curves. We also use the following theorem, given by the author in [4], to establish the main result and obtain an inequality for self-circumference of a plane convex curve with four-fold symmetry, that is, a convex curve $K$ such that $r(K, \theta+\pi / 2)=r(K, \theta)$.

Theorem 1. Assume $K_{1}$ and $K_{2}$ are plane convex bodies with the origin as an interior point. Let $K_{1}^{*}$ denote the polar dual of $K_{1}$. Let $A\left(K_{1}, K_{2}\right)$ denote the mixed area of $K_{1}$ and $K_{2}$. Then

$$
A\left(K_{1}, K_{2}\right) A\left(K_{1}^{*}, K_{2}\right) \geqslant\left[L\left(K_{2}\right)\right]^{2} \text {. }
$$

Equality holds if and only if $K_{1}$ is a circle. 
Proof: Use equalities (5) and (6) to proceed as follows:

$$
\begin{aligned}
4 A\left(K_{1}, K_{2}\right) A\left(K_{1}^{*}, K_{2}\right) & =\left(\int_{0}^{2 \pi} h\left(K_{1}, \theta\right) d s\left(K_{2}, \theta\right)\right)\left(\int_{0}^{2 \pi} \frac{1}{r\left(K_{1}, \theta\right)} d s\left(K_{2}, \theta\right)\right) \\
& \geqslant\left(\int_{0}^{2 \pi} \sqrt{h\left(K_{1}, \theta\right)}\left(\sqrt{r\left(K_{1}, \theta\right)}\right)^{-1} d s\left(K_{2}, \theta\right)\right)^{2} \\
& \geqslant\left(\int_{0}^{2 \pi} d s\left(K_{2}, \theta\right)\right)^{2}=\left[L\left(K_{2}\right)\right]^{2}
\end{aligned}
$$

where we have used the Cauchy-Schwarz inequality and the fact that $h\left(K_{1}, \theta\right) \geqslant$ $r\left(K_{1}, \theta\right)$.

In the above theorem assume $K_{1}=K$ and $K_{2}=B$ where $B$ is the Euclidean unit circle. Then use (3) and (6) to obtain Steinhardt's inequality given in (2).

Assume $K_{1}=K_{2}=K$ and use the isoperimetric inequality given by

$$
L^{2}(K) \geqslant 4 \pi A(K)
$$

and the fact that $A(K, K)=A(K)$ to obtain

$$
A\left(K^{*}, K\right) \geqslant \pi \text {. }
$$

Inequality (16) is a result due to Firey [3]:

The mixed area of a plane convex body and its polar dual is at least $\pi$. In the next section we use Firey's result to obtain a lower bound for self-circumference of sets with four-fold symmetry.

Proof of the Main Result: In Theorem 1 let $K_{1}=K$ and $K_{2}=E^{+}$where $E^{+}$denotes the polar dual of the unit circle $E$ of the Minkowski plane rotated $90^{\circ}$, to obtain

$$
A\left(K, E^{+}\right) A\left(K^{*}, E^{+}\right) \geqslant\left[L\left(E^{+}\right)\right]^{2} .
$$

Then use (5), (6) and (10) to obtain

$$
\sigma_{+}(K, E) \sigma_{+}\left(K^{*}, E\right) \geqslant\left[L\left(E^{+}\right)\right]^{2} .
$$

Since a rotation of $90^{\circ}$ leaves the length invariant, $L\left(E^{+}\right)=L\left(E^{*}\right)$. Thus we obtain the inequality (1) as desired.

In the remainder of this section we use properties of mixed areas and polar duals to obtain additional theorems on self-circumference of convex curves. The following theorem shows that the self-circumference of a plane convex body with four-fold symmetry is at least $2 \pi$. 
THEOREM 2. Let $K$ be a centrally symmetric plane convex body centred at the origin. Assume $r(K, \theta)$ is an equation of the boundary of $K$ in polar coordinates. Assume $r(K, \theta)=r(K, \theta+\pi / 2), 0 \leqslant \theta \leqslant 2 \pi$. That is, $K$ has four-fold symmetry. Then the self-circumference satisfies $\sigma(K) \geqslant 2 \pi$. Equality holds if and only if $K$ is a circle.

Proof: Using the definitions given in (7), and four-fold symmetry, we obtain

$$
\sigma(K)=\int \frac{d s(K, \theta)}{r\left(K, \theta+\frac{\pi}{2}\right)}=\int \frac{d s(K, \theta)}{r(K, \theta)} .
$$

By the property of the polar dual given in (5) and the property of mixed areas given in (6), it follows that

$$
\begin{aligned}
\sigma(K) & =\int \frac{d s(K, \theta)}{r(K, \theta)}=\int h\left(K^{*}, \theta\right) d s(K, \theta) \\
& =2 A\left(K^{*}, K\right) .
\end{aligned}
$$

Firey's result [3] implies $\sigma(K) \geqslant 2 \pi$.

The following can be proved directly from the definition:

Let $K$ be a plane convex body with origin as interior point. Assume $T$ is the isoperimetrix, that is, the polar dual rotated 90 degrees. Then $\sigma_{+}(K, T)=2 A(K)$, where $A(K)$ is the Euclidean area.

If $K$ is a Radon curve, then it coincides with its isoperimetrix. Thus the selfcircumference of a Radon curve is equal to twice its Euclidean area as follows directly from the definitions. We conclude by proving the following theorem, concerning the length of a Euclidean unit circle with respect to a convex curve $K$.

Theorem 3. Let $K$ be a plane convex body. Assume $B$ is the Euclidean unit circle. Then the length of $B$ with respect to $K$ is equal to the Euclidean length of the polar dual of $K$. That is, $\sigma_{+}(B, K)=L\left(K^{*}\right)$.

Proof: By the result of Chakerian given in (12) we obtain

$$
\sigma_{+}(B, K)=\sigma_{-}\left(K^{*}, B^{*}\right)=\sigma_{-}\left(K^{*}, B\right) .
$$

Assuming that the polar dual of $K$ is calculated at the centre of the Euclidean unit circle $B$, it follows that $\sigma_{+}(B, K)=L\left(K^{*}\right)$.

\section{REFERENCES}

[1] G.D. Chakerion, 'Mixed areas and the self-circumference of a plane convex body', Arch. Math. 34 (1980), 81-83. 
[2] H.G. Eggleston, Convexity (Cambridge University Press, Cambridge, 1958).

[3] W.J. Firey, 'The mixed area of a convex body and its polar reciprocal', Israel J. Math. 1 (1963), 201-202.

[4] M. Ghandehari, 'Polar duals of convex bodies', Proc. Amer. Math. Soc. (to appear).

[5] J.J. Schäffer, 'The self-circumference of polar convex disks', Arch. Math. 24 (1973), 87-90.

[6] F. Steinhardt, On distance functions and on polar series of convex bodies, Ph.D. Thesis (Columbia University, 1951).

[7] A.C. Thompson, 'An equiperimetric property of Minkowski circles', Bull. London. Math. Soc. 7 (1975), 271-272.

Department of Mathematics

Naval Postgraduate School

Monterey, CA 93943

United States of America 